\title{
Compton Scattering of a Vortex Light Beam
}

\author{
Mazen Nairat ${ }^{1}$, George Goedecke ${ }^{2}$, David Voelz ${ }^{3}$ \\ ${ }^{1}$ Physics Department, Al Balqa Applied University, Al Salt, Jordan \\ ${ }^{2}$ Physics Department, New Mexico State University, Las Cruces, USA \\ ${ }^{3}$ Klipsch School of Electrical and Computer Engineering, New Mexico State University, Las Cruces, USA
}

Email address:

mazen77@nmsu.edu (M. Nairat), ggoedeck@nmsu.edu (G. Goedecke), davvoelz@nmsu.edu (D. Voelz)

\section{To cite this article:}

Mazen Nairat, George Goedecke, David Voelz. Compton Scattering of a Vortex Light Beam. Optics. Vol. 6, No. 1, 2017 , pp. 1-4. doi: 10.11648/j.optics.20170601.11

Received: May 14, 2017; Accepted: June 1, 2017; Published: July 17, 2017

\begin{abstract}
Energy-momentum conservation laws in Compton scattering are analyzed. The conservation of total angular momentum is applied to a general formula that describes the variation of the light angular momentum. The Compton scattering model of a vortex beam is generalized to describe the momentum exchange beyond the well-known photon wave number shift. The illustrated analysis indicates that the light angular momentum may vary due to Compton scattering.
\end{abstract}

Keywords: Compton Scattering, Light Angular Momentum, Conservation of Momentum

\section{Introduction}

Compton Scattering satisfies conservation both energy and momentum in inelastic collision between a photon and an electron. It states that a certain shift to the linear momentum of the photon is associated with the scattering deflection. The wave number of the photon decreases due to transfer of some its energy and momentum to an initially stationary electron [1]. However; Compton scattering can be generalized to include the conservation of angular momentum too.

Vortex Light beam does have phase singular corresponds to a wave with helical phase structure at the center of the beam the intensity vanishes due to singularity [2]. Orbital angular momentum, of a single photon, is quantized by an integer number called the topological charge of the vortex beam [3]. Vortex light beam carry also spin angular momentum that associates with polarization states. Light angular momentum fundamentally composites of orbital and spin parts.

Several studies have been recently described variation in light angular momentum at Compton scattering in ultra-relativistic considerations $[4,5]$. Moreover, a non-relativistic framework has been implemented in the density matrix theory to inform changes of angular momentum at Compton scattering [6, 7].

This study describes the Compton effect of a vortex light beam which carries both angular and spin angular momentum in form of two components: orbital and spin. We evaluate general analytical expression of Compton scattering for a vortex light. The expression is valid for any axial symmetric beam carries angular momentum such as Laguerre Gaussian and Gaussian Bessel modes [8].

Details are provided on the change of the light angular momentum. Physical explanation as well as geometric interpretation are illustrated. Our analysis is important to understand angular momentum transfer between a photon and an electron or any massive particle.

\section{Theory}

Fundamentally, the angular momentum $(\vec{J})$ of a light beam is composed of orbital part $(\vec{L})$ associated with the azmithual phase at a wave front, and a spin part $(\vec{S})$ associated with the polarization [9]:

$$
\vec{J}=\vec{L}+\vec{S}
$$

In what follows Compton scattering conservation laws are analyzed for a photon vortex beam possessing angular momentum $\vec{J}$ and wave number $k$ colliding with a free electron initially at rest. We assume the electron could possess orbital and spin angular momentum as well [10].

The schematic diagram of the examined Compton scattering is shown at figure 1 which illustrates the scattered photon's wave number and momentum by primed parameters, while the associated momentum of the recoil 
electron is indicated by subscript letter e. The scattering angle $(\theta)$ is defined as the angle between the initial propagation axis and the scattering direction.

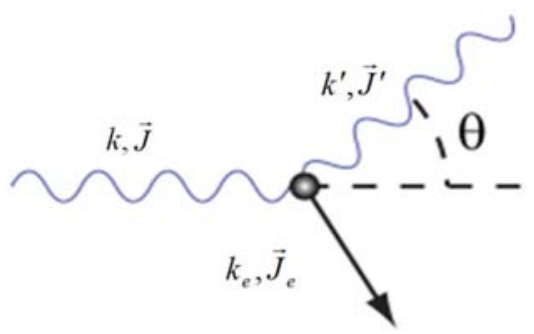

Figure 1. Schematic of Compton scattering with associated parameters.

It is very important to mention that this study particularly considers vortex light beams which are cylindrically symmetric along the propagation axis. Our analysis focuses on three main cases for the vortex light beam: first when it possesses well define orbital angular momentum states, then when it possesses both spin and orbital angular momentum that are separately conserved during free propagation, finally when the orbital and spin angular momentum couple during free propagation. The following sections analyze Compton scattering for each case respectively.

\subsection{Linearly Polarized Light Beam with Orbital Angular Momentum}

A linearly polarized vortex light beam can be modeled as a cylindrically symmetric beam that possesses well-defined orbital momentum states. There are no spin states projected along the symmetric axis in this particular case. The associated linear $(P)$ and orbital momenta $(L)$ are directed along the propagation axis due to the symmetry, so they are linearly proportional to each other by a proportionality constant $(\alpha)$ [11], i.e.:

$$
P / L=k / l=\alpha
$$

where $l$ is the index of the angular momentum state.

Conservation of energy and angular momentum through Compton scattering leads to the following equations:

$$
\begin{gathered}
\alpha_{e}^{2} L_{e}^{2}=\alpha^{2} L^{2}+\alpha^{\prime 2} L^{2}-2 \alpha \alpha^{\prime} L L^{\prime}+2 m c\left(\alpha L-\alpha^{\prime} L^{\prime}\right) \\
L_{e}^{2}=L^{2}+L^{2}-2 L L^{\prime} \cos \theta
\end{gathered}
$$

where $m$ is the rest mass of the electron, and $c$ is the speed of light. Combination of equations (3) and (4) leads to a general formula for Compton scattering of a vortex beam with variation of angular momentum states:

$$
\left(\eta^{2}-1\right) L^{2}+\left(\eta^{\prime 2}-1\right) L^{2}+2 \frac{m c}{\alpha_{e}}\left(\eta L-\eta^{\prime} L^{\prime}\right)=2 L L^{\prime}\left(\eta \eta^{\prime}-\cos \theta\right)
$$

where $\eta$ is the normalized dimensionless ratio of linear to angular momentum, defined by $\eta=\alpha / \alpha_{e}$.

Equation (5) provides many details about the scattering process; however, it is important to check its validity in limiting case before going over the details. In the special case $\eta=\eta=1$, when the ratio of linear momentum to angular momentum never changes through the collision between a photon and an electron, equation (5) reduces to the convential Compton scattering formula:

Generally, when the normalized momentum ratio $\eta=\eta^{\prime}$ is constant through Compton scattering the photon's momentum ratio keeps constant. When there is no change in the photon's orbital momentum due to scattering ( $L=L^{\prime}$ ), equation (5) then reads $\eta-\eta^{\prime}=2 \sin (\theta / 2)$, which indicates a shift in the corresponding linear momentum $\left(P \neq P^{\prime}\right)$. In the meanwhile, if there is no change in the photon's linear momentum, the orbital angular momentum does not vary according to equation (5). But if the linear momentum changes, the angular momentum could still be invariant in Compton scattering. These results are in agreement with previous studies $[5,6]$ concerning the invariance of orbital angular momentum states for forward/backward Compton scattering.

$$
1 / P^{\prime}-1 / P=\frac{1}{m c}(1-\cos \theta)
$$

The ratio of linear to orbital light momenta forms, given in equation (2), can be interpreted in a certain geometrical sense. Light orbital angular momentum is directly proportional to linear momentum in such a way $L$ depends on the orientation of $P$ along of the propagation axis. For instance; the axial momenta at transverse distance $(r)$ away the central propagation axis may be illustrated in simple diagram as shown in Figure 2

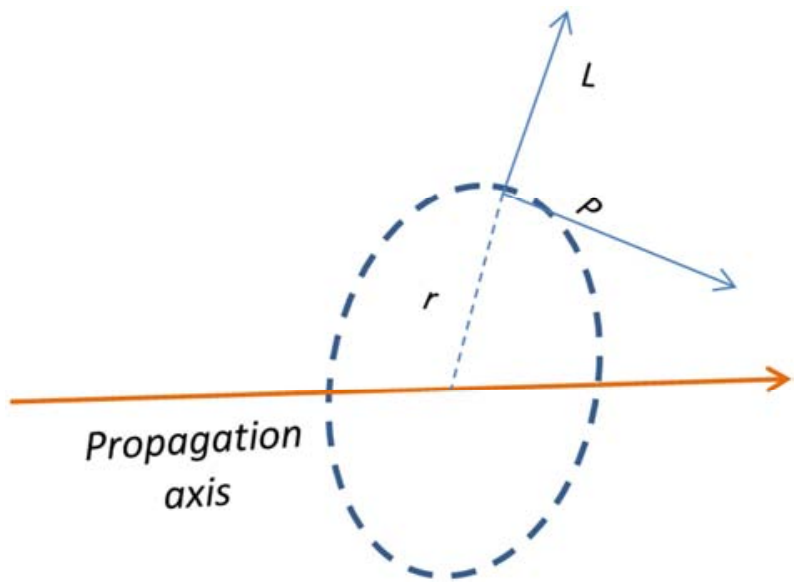

Figure 2a. Schematic of transverse section of a vortex beam with the orientation of the associated momenta. 


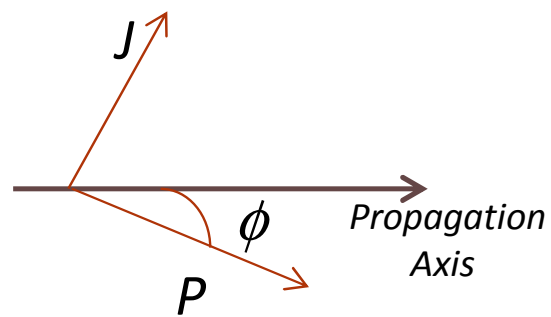

Figure 2b. Side view of the alignment of linear and total angular momentum along a propagation axis.

Figure $2 \mathrm{a}$ represents orientation of both linear and angular momenta of a single photon at transverse section of vortex beam. The alignment of both momenta along the propagation vector is represented in Figure $2 b$.

Geometrically, orbital angular momentum is inclined by $\phi$, which can be defined through the momentum ratio constant $\alpha$ :

$$
\varphi=\arctan (\alpha r)
$$

This interpretation allows one to express the orbital angular momentum as a light characteristic that depends on the axial linear momentum as well as the radius of the beam.
It is easy to use equations (2) and (7) to show that orbital angular momentum vanishes when the axial linear momentum is perfectly aligned with the propagation axis $(\phi$ $=0$ ). Note that singularity point exists at the center of a vortex beam.

\subsection{Circularly Polarized Light Beam with Spin and Orbital Angular Momentum}

A circularly polarized beam possesses spin momentum states along the propagation axis in addition to orbital momentum states. Thus, the ratio of the associated momenta is given by [11]:

$$
\frac{P}{J}=\frac{k}{l+\sigma}=\alpha
$$

where $\sigma$ is the axial spin mode index, analogous to the quantum helicity, which defiantly takes values \pm 1 for lefthanded circularly and right-handed circularly polarized light, respectively.

The Compton scattering formula, Equation (5) may be rewritten in terms of total angular momentum as follows:

$$
\left(\eta^{2}-1\right) J^{2}+\left(\eta^{\prime 2}-1\right) J^{\prime 2}+2 \frac{m c}{\alpha_{e}}\left(\eta J-\eta^{\prime} J^{\prime}\right)=2 J J^{\prime}\left(\eta \eta^{\prime}-\cos \theta\right)
$$

In case there is no coupling of spin and orbital angular momenta, equation (9) could be split two equations describing Compton scattering with variation of each angular momentum component separately, i.e.:

$$
\begin{gathered}
\left(\eta_{l}^{2}-1\right) L^{2}+\left(\eta_{l}^{\prime 2}-1\right) L^{2}+2 \frac{m c}{\alpha_{e}}\left(\eta_{l} L-\eta_{l}^{\prime} L^{\prime}\right)=2 L L^{\prime}\left(\eta_{l} \eta_{l}^{\prime}-\cos \theta\right) \\
\left(\eta_{s}^{2}-1\right) S^{2}+\left(\eta_{s}^{\prime 2}-1\right) S^{\prime 2}+2 \frac{m c}{\alpha_{e}}\left(\eta_{s} S-\eta^{\prime} S^{\prime}\right)=2 S S^{\prime}\left(\eta_{s} \eta_{s}^{\prime}-\cos \theta\right)
\end{gathered}
$$

where $\eta_{s}$ and $\eta_{l}$ refer to the normalized momentum ratios of spin and orbital components respectively. Their combination must be equivalent to the total normalized ratio $\eta=\eta_{l}+\eta_{s}$.

Equations (10) and (11) provide interesting results by examining two opposite cases. One case is Compton scattering of a vortex beam with variation only in the spin momentum part; equation (10) then predicts a change in the wave number of the light beam. The opposite case is Compton scattering of a vortex beam in which spin momentum does not change but orbital momentum does; then equation (11) predicts a shift in the wave number too.

Mathematically, in the case $L=L^{\prime}, S \neq S^{\prime}$, equation (11) reduces to $k-k^{\prime}=2 l \alpha_{e} \sin (\theta / 2)$. In the other case $L \neq L^{\prime}$, $S=S^{\prime}$, equation (12) reduces to $k-k^{\prime}=2 \sigma \alpha_{e} \sin (\theta / 2)$.

Consequently; Compton scattering with variation in either angular momentum part leads to a change in the linear momentum or shift in the wave number. However; the total angular momentum might be unchanged by Compton scattering. Obviously if there is no change in total angular momentum, then equation (9) still predicts a shift in the wave number.

\subsection{Vortex Light Beam with Spin-Orbital Momentum Coupling}

Spin and orbital angular momentum of a vortex beam could be coupled during free propagation [12]. Their coupling is associated with the ratio of total angular momentum to linear momentum that is

$$
\frac{P}{J}=\frac{k}{l \sigma}=\alpha_{c}
$$

Compton Scattering of this case is supposed to be described by total angular momentum $\left(J_{c}\right)$ which associates with the coupling of spin and orbital momenta states: 


$$
\left(\eta_{c}^{2}-1\right) J_{c}^{2}+\left(\eta_{c}^{\prime 2}-1\right) J^{\prime 2}+2 \frac{m c}{\alpha_{e}}\left(\eta_{c} J-\eta_{c}^{\prime} J^{\prime}\right)=2 J J^{\prime}\left(\eta_{c} \eta_{c}^{\prime}-\cos \theta\right)
$$

where $\eta_{c}$ is the normalized momentum coupling ratio: $\eta_{c}=\alpha_{c} / \alpha_{e}$.

Equation (13) clearly shows that regardless of any change in either angular momentum part, a variation in coupling of angular momentum parts leads to a shift in the wave number. Thus; If $L=L^{\prime}$, and $S=S^{\prime}$, but $J_{c} \neq J_{c}^{\prime}$ then $k-k^{\prime}=2 \sigma l \alpha_{e} \sin (\theta / 2)$. Thus, a change in coupling of linear and orbital angular momentum also leads to a shift in the wave number even though no variation any part of light momentum.

\section{Conclusion}

The Compton Scattering momentum formula is generalized to include the conservation of light angular momentum. Specific details are provided to describe unconventional Compton scattering which may cause the light angular momentum to change. A change in any angular momentum part: orbital, spin, or even a coupling between the parts produces a change in wavenumber and linear momentum of the light.

Angular momentum and linear momentum of the light vortex beam are directly proportional with a ratio that determines the momentum alignment with the propagation axis. The normal angular to linear momentum ratio is consistent for vortex light. The conventional Compton scattering, the well-known photon wave number shift, is described at $\alpha=0$.

\section{References}

[1] Compton, Arthur H. "A Quantum Theory of the Scattering of X-Rays by Light Elements". Phys Rev 21 (5): 483-502. (1923).
[2] P. Senthilkumaran, J. Masajada, S. Sato, Interferometry with vortices, Int. J. Opt. (2012).

[3] Progress in Optics, in: M. R. Dennis, K. O'Holleran, M. J. Padgett, E. Wolf (Eds.), Elsevier, (2009).

[4] S. Stock, A. Surzhykov, S. Fritzsche, and D. Seipt 1. "Compton scattering of twisted light: angular distribution and polarization of scattered photons ". Physical Review a 92, 013401 (2015).

[5] I. Ivanov, and V. Serbo. "Scattering of twisted particles: Extension to wave packets and orbital helicity", Phys Rev A, 84 (3): 033804-9.

[6] U. D. Jentschura and V. G. Serbo, "Generation of HighEnergy Photons with Large Orbital Angular Momentum by Compton Backscattering", Phys. Rev. Lett. 106, 013001.

[7] Xiangdong Ji "Deeply virtual Compton scattering". Phys. Rev. D 55, 7114 (1997).

[8] F. Gori, G. Guattari, and C. Padovani, "Bessel-Gauss Beams", Opt. Commun., 64, 491, (1987).

[9] A. O'Neil, I. MacVicar, L. Allen, and M. Padgett. "Intrinsic and extrinsic nature of the orbital angular momentum of a light beam”. Phys Rev Let 88 (5): 053601-4, (2002).

[10] L. Allen, M. W. Beijersbergen, R. J. C. Spreeuw, and J. P. Woerdman "Orbital angular momentum of light and the transformation of Laguerre-Gaussian laser modes" Phys. Rev. A 45, 8185. (1992).

[11] M. Padgett, and L. Allen. "Light with a twist in its tail", Contemporary Physics, 2000, 41 (5), (2000).

[12] L. Allen, V. Lembessis, and M. Babiker "Spin-orbit coupling in free-space Laguerre-Gaussian light beams" Phys. Rev. A 53, R2937, (1996). 